FÁBIO SAKAE KUTEKEN ${ }^{1}$

Carmen lucia Penteado Lancellotti ${ }^{2}$

Heizabet Salomão AbDalla Ayroza Ribero 3

JOSÉ MENDES ALDRIGH ${ }^{4}$

Paulo Augusto Ayroza Galvão Ribero ${ }^{5}$

\title{
Expressão de mediadores neurotróficos e pró-inflamatórios na endometriose de reto e sigmoide
}

\author{
Expression of neurotrophic and inflammatory mediators in \\ rectosigmoid endometriosis
}

Artigo Original

\begin{abstract}
Palavras-chave
Fator de necrose tumoral alfa Fator de crescimento neural

Neuropeptideo Y

Peptídeo intestinal vasoativo

Endometriose

Doenças do colo sigmoide

Doenças retais

Fibras nervosas

Keywords

Tumor necrosis fator-alpha

Nerve growth factor

Neuropeptide $Y$

Vasoactive intestinal peptide

Endometriosis

Sigmoid diseases

Rectal diseases

Nerve fibers
\end{abstract}

Correspondência

Fóbio Sakae Kuteken

R. Cesário Mota Junior, 61, 13 andar - Vila Buarque

CEP: 01221-020

São Paulo (SP), Brasil

Recebido

$18 / 01 / 12$

Aceito com modificacões

$18 / 10 / 12$

\section{Resumo}

OBJETIVO: Avaliar a expressão de mediadores neurotróficos (NGF, NPY E VIP) e pró-inflamatórios (TNF-a) em fragmentos de reto e sigmoide comprometidos por endometriose. MÉTODOS: Foram selecionadas 24 pacientes submetidas ao tratamento cirúrgico de endometriose de reto e sigmoide com técnica de ressecção segmentar, seguido de anastomose mecânica término-terminal, com grampeador circular, no período de janeiro de 2005 a dezembro de 2007. Neste estudo incluímos mulheres no menacme que se submeteram a tratamento cirúrgico por endometriose profunda infiltrativa com acometimento do reto e sigmoide, atingindo o nível da camada muscular, submucosa ou mucosa. Para o grupo de estudo foram utilizados 24 fragmentos de reto e sigmoide com endometriose confirmada histologicamente, sendo um fragmento de cada uma das 24 pacientes selecionadas. Para o grupo controle, utilizou-se um fragmento da margem distal da ressecção, denominado anel de anastomose, de cada uma das 24 pacientes selecionadas e incluídas no estudo. As amostras foram agrupadas em blocos de Tissue Micro Array (TMA) e submetidas à reação imunoistoquímica para avaliar a expressão do fator de necrose tumoral alfa (TNF-a), do fator de crescimento neural (NGF), do neuropeptídeo Y (NPY) e do peptídeo intestinal vasoativo P (VIP), e posterior análise semiquantitativa da imunomarcação por meio da leitura da densidade ótica relativa (DO). RESULTADOS: Observou-se maior densidade ótica relativa da imunomarcação para TNF-a e NGF no grupo de estudo (amostras com endometriose intestinal), $D O=0,01$, respectivamente, para as duas proteínas $(p<0,05)$, em relação aos controles sem endometriose. Não houve diferença estatística na densidade ótica da imunomarcação do NPY e VIP. CONCLUSÃO: Identificou-se o aumento da imunomarcação dos anticorpos TNF-a e NGF em fragmentos de reto e sigmoide comprometidos por endometriose em relação aos controles livres da doença. Não identificamos diferença estatística na imunomarcação das proteínas NPY e VIP.

\section{Abstract}

PURPOSE: To evaluate the expression of neurotrophic (NGF, NPY and VIP) and pro-inflammatory (TNF-a) mediators in the rectum and sigmoid fragments compromised by endometriosis. METHODS: Twenty-four patients were selected to undergo surgical treatment of endometriosis of the rectum and sigmoid colon with a segmental resection technique, followed by end-toend anastomosis with a circular stapler from January 2005 to December 2007. The study included premenopausal women who underwent surgical treatment for deep endometriosis infiltrating the rectum with involvement of the rectum and sigmoid, reaching the level of the muscle layer, submucosa or mucosa. Twenty-four rectum and sigmoid fragments with histologically confirmed endometriosis, one from each of the 24 selected patients, were used for the study group. For the control group, we used a fragment of the distal resection margin called anastomosis ring from each of the 24 patients enrolled in the study. Samples were grouped into Tissue Micro Array (TMA) blocks and subjected to immunohistochemistry to evaluate the expression of tumor necrosis factor alpha (TNF-a), nerve growth factor (NGF), neuropeptide Y (NPY) and P vasoactive intestinal peptide (VIP), followed by semiquantitative analysis of immunostaining by reading the relative optical density (OD). RESULTS: There was higher optical density relative to TNF-a immunostaining and NGF in the study group (samples with intestinal endometriosis), $\mathrm{DO}=0.01$, for the two proteins, respectively $(\mathrm{p}<0.05)$, compared to controls without endometriosis. There was no statistically significant difference in the optical density of immunostaining of NPY and VIP. CONCLUSION: We identified increased immunostaining of TNF-a antibodies and fragments of NGF in the rectum and sigmoid compromised by endometriosis compared to disease-free controls. We did not identify any statistical difference in immunostaining of NPY and VIP proteins.

Trabalho realizado no Departamento de Obstetrícia e Ginecologia da Faculdade de Medicina da Santa Casa de São Paulo São Paulo (SP), Brasil

'Setor de Endoscopia Ginecológica e Endometriose e Departamento de Obstetrícia e Ginecologia, Santa Casa de Misericórdia de São Paulo - SCMSP - São Paulo (SP), Brasil.

2Departamento de Anatomia Patológica, Faculdade Ciências Médicas da Santa Casa de Misericórdia de São Paulo - FCMSCSP. São Paulo (SP), Brasil.

${ }^{3}$ Setor de Endoscopia Ginecológica e Endometriose do Departamento de Obstetrícia e Ginecologia, Santa Casa de Misericórdia de São Paulo - SCMSP - São Paulo (SP), Brasil.

4Departamento de Obstetrícia e Ginecologia, Faculdade Ciências Médicas da Santa Casa de Misericórdia de São Paulo FCMSCSP - São Paulo (SP), Brasil.

${ }^{5}$ Setor de Endoscopia Ginecológica e Endometriose do Departamento de Obstetrícia e Ginecologia, Santa Casa de Misericórdia de São Paulo - SCMSP - São Paulo (SP), Brasil. 


\section{Introdução}

A endometriose é uma doença de etiologia incerta e múltiplas variáveis podem contribuir para sua gênese. Trata-se de moléstia que acomete 10 a $15 \%$ da população feminina em idade reprodutiva e estima-se que, dentre as portadoras de dor pélvica crônica, a doença possa ocorrer em até 60 ou $70 \%$ das pacientes ${ }^{1,2}$. Dentre as diversas formas de manifestação da doença, a endometriose infiltrativa profunda chama atenção pela sintomatologia intensa e pela atividade celular traduzida em proliferação tecidual. A endometriose profunda é definida como a presença de células endometriais, glândulas e ou estroma, com infiltração maior que $5 \mathrm{~mm}$ abaixo do peritônio ${ }^{3}$. A endometriose profunda habitualmente localiza-se nos ligamentos uterossacrais, ovários, retossigmoide, vagina e bexi$\mathrm{ga}^{4}$. A endometriose intestinal é uma das formas mais graves da doença, podendo acometer entre 5,3 e 12\% das mulheres portadoras de endometriose 5 .

A possível relação entre a presença de nervos simpáticos e parassimpáticos e o desenvolvimento dos nódulos de endometriose no septo retovaginal foi descrita há pouco mais de uma década ${ }^{6}$. Não obstante, diversos aspectos ainda permanecem pouco claros e não esclarecidos pelos estudos conduzidos neste período. No intuito de esclarecer estes mecanismos, alguns autores concentraram suas pesquisas na relação da endometriose com o processo inflamatório que envolve os nervos da região acometida ${ }^{7,8}$, e observaram que as mulheres que apresentavam maiores pontuações em escalas de dor também apresentavam invasão intra e perineural por focos de endometriose ${ }^{8}$.

Embora seja certo que níveis elevados de citocinas são um resultado direto da doença, ainda não está claro se a secreção dessas proteínas pró-inflamatórias e a associação com as células do sistema imunológico no microambiente peritoneal contribui para a cascata de eventos que resultam no estabelecimento da dor e na progressão da endometriose. Neste contexto, uma citocina que ganhou atenção especial na fisiopatologia da endometriose foi o fator de necrose tumoral alfa $(\mathrm{TNF}-\mathrm{a})^{9}$. O TNF-a é uma citocina pró-inflamatória com uma série de efeitos que dependem da quantidade produzida, da sua localização tecidual, da atividade local, do meio hormonal e de outras citocinas ${ }^{9}$. O TNF-a desempenha um papel importante em vários processos fisiológicos e patológicos, incluindo a proliferação celular, diferenciação, apoptose, modulação da resposta imune e indução da inflamação ${ }^{9,10}$.

O fator de crescimento neural (NGF) é uma proteína neurotrófica que modula o crescimento e a sobrevivência de diversos neurônios periféricos e centrais. Os efeitos do NGF sobre o crescimento e sobrevivência neuronal são mediados por um receptor de alta afinidade na superfície celular, receptor TRKA (Receptor-A de tirosina quinase) e um receptor neurotrófico de baixa afinidade (p75), podendo sinalizar suas ações neuroprotetoras por meio do TRKA e desencadear apoptose em algumas células a partir do receptor $\mathrm{p} 75^{11,12}$. O NGF é um importante mediador multifuncional, podendo mediar a dor e aumentar a regulação das fibras nervosas associadas com as áreas inflamadas, pois atua diretamente na degranulação de mastócitos, ativando os receptores de dor e estimulando a sensação de dor ${ }^{13}$.

Recentemente, alguns autores demonstraram a presença de fibras nervosas específicas no tecido endometrial, com associação entre a densidade de fibras nervosas no endométrio e a gravidade da dor. Estas mesmas fibras nervosas, simpáticas e parassimpáticas, foram encontradas nas lesões endométrioticas e no miométrio ${ }^{14}$.

Baseados nestes achados descritos e nas evidências do desenvolvimento de endometriose em locais ricamente inervados, postulou-se a hipótese da infiltração nos nervos como parte da fisiopatologia desta doença ${ }^{15}$. Assim sendo, e considerando-se que a inervação intestinal é maior do que na maioria dos outros órgãos pélvicos, a endometriose profunda teria uma facilidade de infiltrar-se no intestino através dos seus plexos nervosos, induzindo a dor intensa, de forma única e não similar ao observado em outros órgãos. Este pensamento é confirmado por alguns estudos, nos quais demonstrou-se que a densidade total das fibras nervosas dos fragmentos de endometriose intestinal foi seis vezes maior que nas lesões de endometriose obtidas em outros sítios ${ }^{16}$.

Os estudos que buscaram avaliar as proteínas possivelmente associadas ao aumento do tecido neural nas lesões de endometriose identificaram, dentre vários outros, o peptídeo intestinal vasoativo (VIP) e o neuropeptídeo Y (NPY) como possíveis marcadores da proliferação dos nervos viscerais pélvicos ${ }^{17}$. O VIP é um neuropeptídeo neurotrófico que pode estimular a mitose, promovendo inflamação neuronal e aumentando a sobrevivência dos neurônios parassimpáticos precursores. Pode contribuir para o aumento da indução da lesão neural no desenvolvimento de inflamação nos neurônios sensoriais e na medula espinhal e pode ser relevante para o surgimento de terminações centrais anormais dos neurônios sensitivos, após lesões dos nervos periféricos ${ }^{17}$. Já o NPY é um peptídeo neurotrófico dos nervos simpáticos do sistema nervoso autonômico da pelve. Está presente em grande quantidade na cérvix uterina, cúpula vaginal e ligamentos uterossacros ${ }^{18}$.

No entanto, a relação entre nervos e lesão de endometriose ainda é pouco clara e advoga-se que as lesões de endometriose seguiriam a rota dos nervos, porque esta seria uma zona de baixa resistência à proliferação 
da doença, e a camada muscular ofereceria um obstáculo para a proliferação ${ }^{8}$.

Em nosso serviço, demonstrou-se, por meio da imunoistoquímica dos anticorpos NPY e VIP, maior número das fibras nervosas nos ligamentos uterossacrais acometidos pela endometriose ${ }^{19}$, confirmando outros achados que sugerem não só a maior proliferação neuronal destes tecidos, mas também a possível associação deste fenômeno com a gênese da doença ${ }^{17-20}$.

Baseados nos dados expostos e buscando ampliar a compreensão com os fenômenos envolvidos na gênese da doença e na fisiopatologia da dor associada à endometriose, entendemos ser adequado analisar a presença destes fatores, TNF-a, NGF, NPY (nervos simpáticos) e VIP (parassimpáticos), em segmentos de reto e sigmoide comprometidos por endometriose.

\section{Métodos}

Trata-se de estudo transversal realizado no setor de Endoscopia Ginecológica e Endometriose do Departamento de Obstetrícia e Ginecologia (DOGI) da Irmandade da Santa Casa de Misericórdia de São Paulo (ISCMSP). O estudo foi realizado após a aprovação do Comitê de Ética em Pesquisa em Seres Humanos desta instituição.

Foram selecionadas 24 pacientes submetidas ao tratamento cirúrgico de endometriose de reto e sigmoide com técnica de ressecção segmentar, seguido de anastomose mecânica término-terminal, com grampeador circular, no período de janeiro de 2005 a dezembro de 2007. Incluímos neste estudo mulheres no menacme que se submeteram a tratamento cirúrgico por endometriose profunda infiltrativa com acometimento do retossigmoide, atingindo o nível da camada muscular, submucosa ou mucosa. As pacientes não podiam ter usado medicações hormonais, análogo de $\mathrm{GnRH}$ e anti-inflamatórios por pelo menos três meses antes da cirurgia. Foram excluídas as mulheres que apresentavam na avaliação histológica reto e sigmoide com acometimento apenas da serosa e gordura pericólica, pacientes que tinham algum tipo de doença crônica, neoplasia maligna e aquelas que faziam uso regular de medicamentos, hormônios e anti-inflamatórios.

Foram utilizados para o grupo de estudo 24 fragmentos de reto e sigmoide com endometriose confirmada histologicamente, sendo um fragmento de cada uma das 24 pacientes selecionadas. Para o grupo controle, utilizou-se um fragmento da margem distal da ressecção, denominado anel de anastomose, de cada uma das 24 pacientes selecionadas e incluídas no estudo.

$O$ estadiamento da endometriose foi feito pela classificação revisada da American Society for Reproductive Medicine ${ }^{20}$ e todas as nossas pacientes apresentaram escore superior a 40 , sendo classificadas como estádio IV.
As pacientes estudadas encontravam-se entre 25 anos e 48 anos de idade, com média de 35,5 anos e desvio padrão (DP) de 5,4.

Após fixação em solução de formaldeído tamponado a $10 \%$, o material foi encaminhado ao Serviço de Anatomia Patológica da ISCMSP. Realizou-se inicialmente a análise macroscópica dos espécimes; a seguir, houve a desidratação em álcool etílico, clareados pelo xilol e embebidos em parafina, para confecção dos blocos de parafina. Os blocos foram cortados por meio de micrótomo calibrado para cortes de $4 \mu \mathrm{m}$ de espessura. Os cortes histológicos foram corados pelo método de hematoxilina-eosina (HE) e a leitura foi realizada em microscópio óptico comum. Os casos foram avaliados por examinador experiente e os laudos foram emitidos de acordo com a padronização utilizada pela instituição. Todos os casos apresentavam endometriose e foram selecionados para a confecção do arranjo tecidual em matriz, consagradamente conhecido como tissue microarray (TMA).

Microarranjo tecidual (tissue microarray - TMA) e imunoistoquímico

A técnica de TMA utilizada no presente estudo foi realizada no laboratório de Consultoria em Patologia Botucatu-São Paulo. Para tal, empregou-se a marcação do local de interesse de estudo no bloco, com visualização prévia da lesão na lâmina. Desta forma, obtivemos as áreas marcadas, visando à confecção do TMA.

A técnica imunoistoquímica utilizada no presente estudo foi realizada seguindo a técnica-padrão do serviço, com controles negativos e os controles positivos de cada anticorpo para validar a imunomarcação.

Os anticorpos primários utilizados nas reações imunoistoquímicas foram: fator de necrose tumoral alfa (TNF-a): buman t-alpha affinity purified polyclonal Ab, goat Igg (RnDSystems), fator de crescimento neural (NGF): polyclonal rabbit anti-buman ngf (Santa Cruz Biotechnology, Santa Cruz, California), peptídeo intestinal vasoativo (VIP): polyclonal rabbit anti-buman Vip (Chemicon, Temecula, California) e neuropeptídeo Y (NPY): polyclonal rabbit anti-buman npy (Chemicon, Temecula, California), nas diluições 1/500, 1/5.000, $1 / 5.000$ e $1 / 5.000$, respectivamente.

Os cortes foram incubados com os anticorpos primários preparados em solução previamente otimizada de TNF-a, NGF, VIP e NPY. Para visualização da reação, os cortes foram tratados com substrato cromogênico (diaminobenzidina a $60 \mathrm{mg} \%$ em PBS associado a $1,5 \mathrm{~mL}$ de peróxido de hidrogênio a 20 volumes), por cinco minutos, a $37^{\circ} \mathrm{C}$. Os cortes foram contra corados com hematoxilina de Harris, seguindo-se desidratação e montagem em entellan com lamínula (Figuras 1 e 2). 
Avaliação semiquantitativa da imunoistoquímica

Dos cortes histológicos obtidos pelo TMA, foram feitas lâminas sem a coloração de fundo (hematoxilina) para facilitar a avaliação da imunomarcação dos fatores estudados por meio da leitura da densidade ótica relativa. A avaliação imunoistoquímica dos fatores NGF, NPY, VIP E TNF-a foi então realizada utilizando o sistema MCID de análise densitométrica digital (InterFocus Imaging Ltd., Linton, England). Este método mede a intensidade da imunomarcação em tons de cinza, gerando um valor numérico da densidade ótica relativa, comparado a um padrão obtido a partir da leitura de uma lâmina do mesmo fragmento tecidual, corada somente com anticorpo primário. Foram obtidos o valor numérico final positivo ou negativo e a diferença entre a leitura do padrão e a lâmina de estudo corada com anticorpos primários e secundários. Obtivemos, assim, números absolutos da densidade ótica relativa, que não representam escalas ordinais numéricas, mas sim uma intensidade luminosa comparada a uma densidade padrão do sistema (Figura 2).

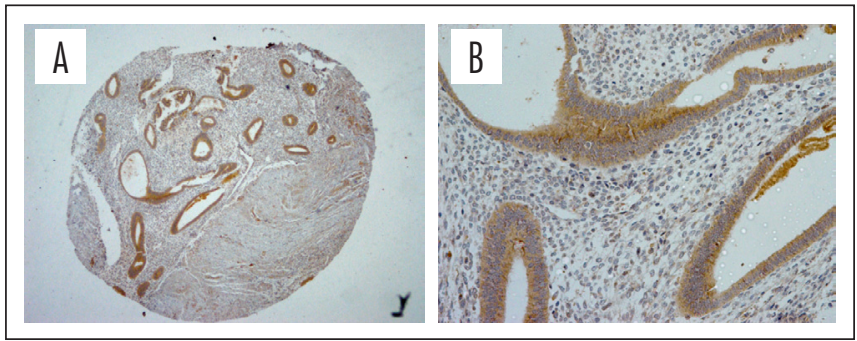

Figura 1. A: Microfotografia da imunoistoquímica para o neuropeptídeo $Y$ (NPY) - polyclonal rabbit anti-human npy. aumento 100x. B: Lâmina com imunoistoquímica para o neuropeptídeo Y (NPY) - polyclonal rabbit anti-human NPY. aumento 400x

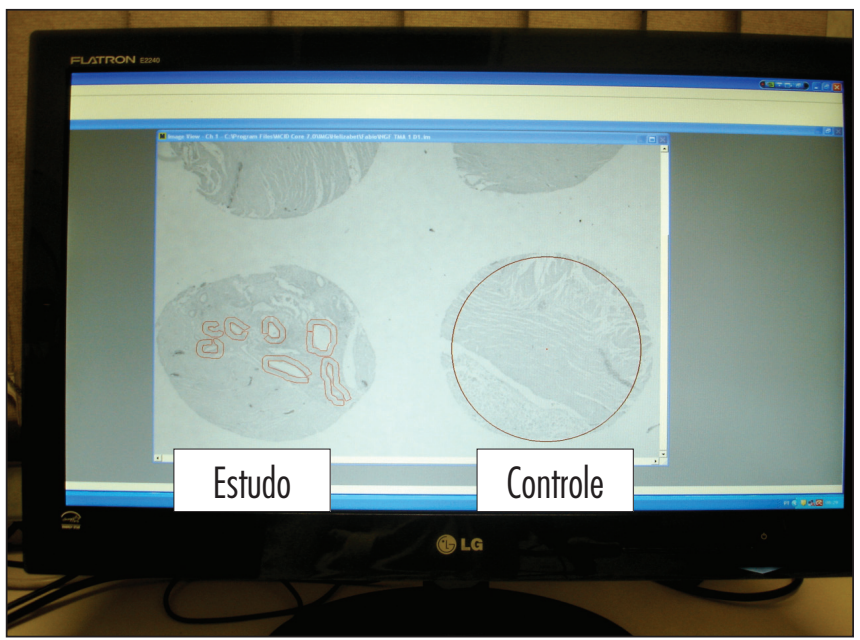

Figura 2. Leitura da densidade óptica relativa de um caso do grupo de estudo e controle, coradas com NGF (polyclonal rabbit anti-human ngf), marcadas dentro das áreas escolhidas em vermelho.

\section{Análise Estatística}

O cálculo do tamanho da amostra foi realizado considerando-se o erro $\mathbf{a}=\mathbf{a} 0,05$ e poder da amostra $\geq 80 \%$, e chegou-se a um tamanho mínimo da nossa amostra de 24 indivíduos em cada grupo. Utilizou-se o programa Microsoft Excel 2007 para arquivamento dos dados e o programa SPSS for Windows 11.0 para as análises estatísticas. Empregou-se o teste de Kolmorgorov-Smirnov para avaliar a distribuição dos resultados obtidos e, após evidenciar-se a distribuição normal dos dados estudados, optou-se por utilizar para a expressão das variáveis semiquantitativas a média, desvio- padrão e intervalo de confiança.

Para as análises estatísticas das variáveis semiquantitativas utilizamos o teste t-pareado, adequado para nosso estudo por ser um comparativo pareado no qual cada paciente fornece um par de material para serem analisados.

\section{Resultados}

A análise descritiva de nossos resultados demonstrou que a média da densidade ótica relativa da imunoistoquimica do TNF-a foi de $0,01( \pm 0,03)$ no grupo de estudo, e $0,01( \pm 0,02)$ no grupo controle. A imunomarcação do NGF apresentou média da densidade ótica relativa de $0,01( \pm 0,02)$ no grupo de estudo, e nos controles a média da densidade ótica relativa foi $-0,01( \pm 0,02)$. Os resultados da imunomarcação do NPY demonstraram média de 0,04 $( \pm 0,04)$ e $0,02( \pm 0,04)$, respectivamente, nos grupos de estudo e controle. Quanto ao VIP, observou-se média de $0,01( \pm 0,02)$ no grupo de estudo e $0,01( \pm 0,02)$ no grupo controle (Figura 3).

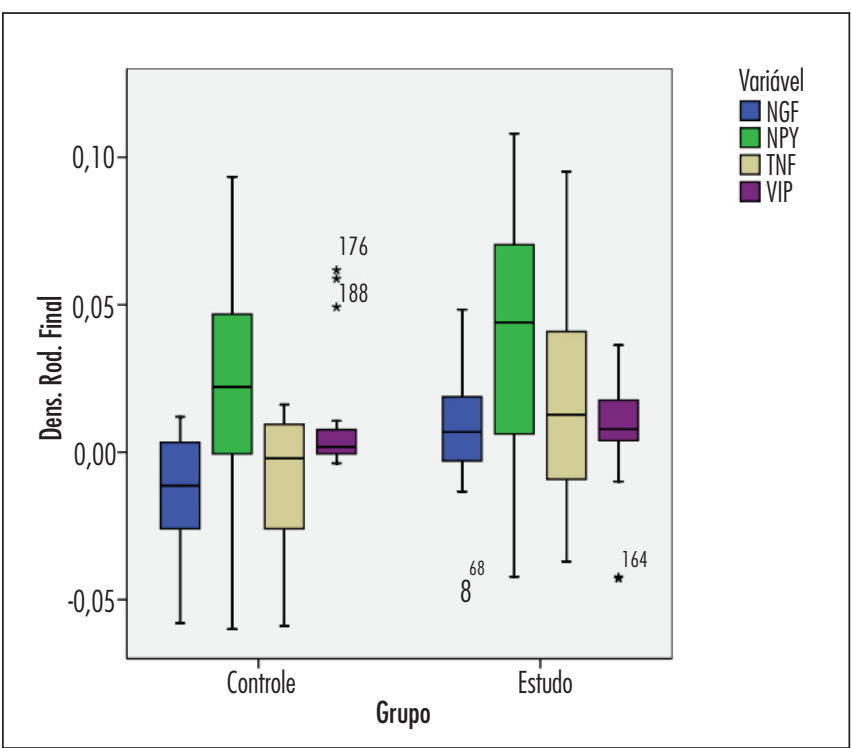

Figura 3. Densidade ótica relativa nos fragmentos de reto e sigmoide comprometidos com endometriose (grupo de estudo) e nos controles livres da doença (grupo controle). 
Tabela 1. Análise da densidade ótica relativa (DO) dos fatores TNF-a, NGF, NPY e VIP em fragmentos de reto e sigmoide com endometriose (grupo de estudo) e nos anéis de anastomose livres da doença (grupo controle)

\begin{tabular}{lccccc}
\hline & \multicolumn{2}{c}{ Estudo } & \multicolumn{2}{c}{ Controle } & \multirow{2}{*}{ Valor $p$} \\
\cline { 2 - 5 } & $\begin{array}{c}\text { Média da } \\
\text { DO (DP) }\end{array}$ & IC95\% & $\begin{array}{c}\text { Média da DO } \\
\text { (DP) }\end{array}$ & IC95\% & \\
\hline TNF-a & $0,01(0,03)$ & $0,00-0,03$ & $-0,01(0,02)$ & $-0,02--0,00$ & $0,002^{*}$ \\
NGF & $0,01(0,00)$ & $-0,03-0,02$ & $-0,01(0,02)$ & $-0,02--0,04$ & $<0,001^{\star}$ \\
NPY & $0,04(0,04)$ & $0,02-0,06$ & $0,02(0,04)$ & $0,01-0,04$ & 0,08 \\
VIP & $0,01(0,02)$ & $-0,01-0,00$ & $0,01(0,02)$ & $0,00-0,02$ & 0,78 \\
\hline
\end{tabular}

* teste † pareado; TNF-a: Fator de necrose tumoral a; NGF: Fator de crescimento neural; NPY: Neuropeptídeo Y; VIP: Peptídeo vasoativo; DP: Desvio Padrão; IC: Intervalo de confiança.

A análise estatística de nossos resultados demonstrou que a imunomarcação do TNF- $a(p=0.02)$ e do NGF $(\mathrm{p}<0.001)$ foi maior nos fragmentos intestinais com endometriose em comparação aos controles livres da doença. Por outro lado, a análise estatística da densidade ótica relativa da imunomarcação dos fatores NPY e VIP não demonstrou diferença entre os segmentos intestinais acometidos por endometriose e os controles livres da doença (Tabela 1).

\section{Discussão}

A fisiopatologia da dor na endometriose ainda é incerta, mas alguns dados nos fazem acreditar que existe um aumento da densidade de fibras nervosas associado ao processo inflamatório destas fibras ${ }^{19,21}$. Este seria um dos mecanismos responsáveis pelo início, manutenção e diminuição do limiar da dor, gerando intensa dor e hiperalgesia nas pacientes acometidas por tal enfermidade ${ }^{21}$. Se a teoria da implantação for verdadeira, as fibras nervosas encontradas nas placas de endometriose podem ter origem nos progenitores de fibras nervosas da camada funcional do endométrio ou no crescimento de fibras nervosas locais devido à secreção de NGF e ao aumento da expressão de seus receptores ${ }^{22,23}$.

Sugere-se a existência de diversos tipos de fibras nervosas na camada basal e na camada funcional do endométrio, sendo maior a expressão destas fibras nas pacientes com endometriose ${ }^{23}$. Esta característica, somada a outras alterações do endométrio, como a diminuição da apoptose, a presença de polimorfismo dos receptores da progesterona e a diminuição da vigilância imunológica, proporciona condições facilitadoras para o desenvolvimento da endometriose ${ }^{9,11,24}$. Soma-se também o aumento da expressão do NGF e do TNF- $a^{21-23}$, entre outras citocinas secretadas pelos macrófagos, que facilitariam a implantação e a progressão das células endometrióticas no peritônio pélvico e/ou serosa intestinal, originando os implantes profundos peritoneais e a infiltração da parede intestinal ${ }^{10,11}$.
O aumento da expressão do TNF-a e NGF no fluido peritoneal de pacientes com endometriose foi demonstrado previamente ${ }^{24,25}$. Em nosso estudo, demonstramos que a presença da proteína TNF-a foi mais frequente nos segmentos intestinais comprometidos por endometriose em comparação aos segmentos livres da doença em uma mesma mulher. Este achado confirma a hipótese da interferência do TNF-a na progressão e na manutenção da doença endometriótica intestinal, pois os segmentos intestinais livres de endometriose, embora muito próximos à área comprometida, apresentaram menor expressão desta proteína quando comparados com a área adjacente comprometida por endometriose ${ }^{25}$. Note-se que nosso controle constituiu-se de fragmentos de intestino da mesma região anatômica (reto e sigmoide) dos mesmos indivíduos, diminuindo assim o possível viés da interferência de fatores genéticos na gênese da maior expressão dos fatores estudados.

Baseados na premissa da elevada presença da TNF-a nos segmentos de reto e sigmoide comprometidos por endometriose, seria possível sugerir terapias com uso de drogas com efeito supressor do TNF-a nas pacientes acometidas pela endometriose intestinal. De fato, tal hipótese terapêutica ainda não se confirmou efetiva, pois não dispõe da droga ideal que permita o bloqueio seletivo do TNF-a sem interferir em outras funções vitais da vida celular ${ }^{26}$.

No que se refere ao desenvolvimento e à proliferação neuronal, ressalta-se que estes processos são resultado da ação coordenada de alguns fatores neurogênicos, dentre os quais o NGF apresenta uma ação efetiva. Neste sentido, é importante enfatizar a elevada expressão desta proteína nos nódulos de adenomiose, bem como sua possível relação com a hiperalgesia observada por essas pacientes ${ }^{15,16}$. De fato, a invasão ao longo e em torno dos nervos determina o substrato anatômico para o quadro doloroso observado nestas pacientes ${ }^{15}$.

Considerando-se algumas similaridades entre a adenomiose e a doença endometriótica, sugere-se, complementarmente, que a ação neurotrófica da NGF teria um papel preponderante na gênese da neurite observada em pacientes com endometriose ${ }^{24}$. De forma oposta a este raciocínio, demonstrou-se que a presença de NGF no fluido peritoneal de pacientes com endometriose não se mostrou associada com os diferentes níveis de $\operatorname{dor}^{25}$. Parece-nos, portanto, que à luz dos conhecimentos atuais não se pode traçar um raciocínio similar para a ação desta proteína no fluido peritoneal e no interior das lesões de endometriose profunda.

Observamos a associação da presença da NGF aos fragmentos de tecido intestinal comprometidos por endometriose. Apesar não termos avaliado a correlação 
da presença do NGF com o quadro álgico vivenciado pelas pacientes, sabe-se de estudos anteriores que a dismenorreia está presente em $92 \%$ das portadoras de endometriose profunda, e a dispareunia de profundidade, em $96 \%$ destas pacientes ${ }^{27}$. Fundamentalmente, a expressão da NGF nas pacientes com endometriose é significativamente mais notável do que naquelas sem a doença ${ }^{15}$. Esses dados apoiam a hipótese de que outros fatores também desempenham um papel na geração da dor e encontram suporte nos achados de nosso estudo, que evidenciaram a maior expressão do NGF somente na área do intestino comprometido por endometriose. Assim, não somente o fluido peritoneal não demonstra elevação do $\mathrm{NGF}^{24,25}$, mas os tecidos adjacentes aos focos de endometriose intestinal também demonstram o mesmo comportamento. Outros autores sugeriram que o aumento da expressão do NGF também estaria relacionado a um estímulo à angiogênese, sendo um importante fator para a manutenção da doença ${ }^{18}$. Com as ações descritas anteriormente, entendemos que estas células endometriais ectópicas têm potencial para o desenvolvimento errático, produzindo um processo inflamatório intenso, o qual levaria a um feedback positivo na cascata de eventos que podem disseminar e perpetuar a proliferação neuronal e dos implantes de endometriose.

Com relação aos outros fatores aqui estudados, NPY e VIP, sabe-se que representam marcadores específicos da inervação simpática e parassimpática tecidual $^{17,21}$. Relata-se também a elevada presença (hiperinervação) dessas proteínas nos implantes endometrióticos em reto, sigmoide e apêndice, e também em outros sítios de endometriose profunda infiltrativa como ligamento uterossacro e região retrocervical ${ }^{16,19}$. Esta hiperinervação seria decorrente da invasão neuronal pelos focos de endometriose, que levaria a uma injúria destes nervos e, em reposta, novas terminações nervosas surgiriam a partir do estímulo das proteínas NPY e VIP ${ }^{16}$.

Com base em estudos anteriores realizados em nosso serviço ${ }^{19}$ e nos achados da literatura, esperava-se que a imunomarcação dos anticorpos NPY e VIP nas lesões de endometriose intestinal fosse aumentada em relação ao intestino sadio. Esta hipótese, no entanto, não foi confirmada neste estudo, que demonstrou a presença similar dos NPY e VIP nos segmentos de reto e sigmoide com endometriose em comparação àqueles sem a doença. Questionamos, portanto, se a hiperexpressão destes fatores no intestino, descrita previamente por outros autores $^{15}$, não ocorreria de forma homogênea em todo o intestino - comportamento inverso ao observado pelos outros fatores aqui estudados.

Contribui para esta conclusão a diminuição da densidade dos nervos simpáticos observada na camada muscular e na mucosa intestinal de áreas adjacentes às lesões de endometriose, em comparação ao observado nos tecidos saudáveis coletados na margem da ressecção intestinal realizada nas mesmas pacientes ${ }^{28}$. Sugere-se também que a infiltração retroperitoneal da endometriose seguiria estritamente o caminho dos nervos simpáticos, enquanto os nervos parassimpáticos seriam ocasionalmente envolvidos nesta infiltraçãa ${ }^{18}$. Isto nos faz pensar que de forma similar, na invasão intestinal, poderia ocorrer a infiltração preferencial pela inervação simpática. Este pensamento é coerente com os achados de nosso estudo, que não evidenciou alterações da presença do VIP nos segmentos intestinais com endometriose em comparação aos fragmentos sem a doença.

Analisando de forma conjunta os achados de nosso estudo e tentando traçar um paralelo com os achados da literatura, encontramos alguns pontos controversos acerca da expressão ou presença de alguns dos fatores estudados no intestino. Parece-nos relevante a concordância acerca da associação da presença dos fatores TNF-a e NGF dos fragmentos de reto e sigmoide comprometidos por endometriose. Não obstante, causa-nos surpresa o fato de não haver maior presença de VIP e NPY nos tecidos de reto e sigmoide comprometidos por endometriose em comparação com as áreas saudáveis do intestino desses mesmos indivíduos ${ }^{16,18,19,28,29}$.

A escolha do nosso grupo controle como sendo a amostra intestinal nos anéis de anastomose distais da mesma paciente pode não ter sido a ideal, visto que a proximidade com o foco de endometriose pode ter influenciado nos resultados obtidos quanto ao NPY e VIP. No entanto, amostras intestinais provenientes de outras enfermidades, como câncer intestinal, doença de Crohn e outras, também poderiam ser questionadas, pois tratam-se de doenças cuja fisiopatologia, sabidamente inflamatória, poderia interferir na presença destes fatores.

Novos estudos são necessários para elucidar o comportamento da fibras nervosas na fisiopatologia nas lesões da endometriose intestinal. Talvez a correlação entre tipos de fibras nervosas do endométrio de mulheres com endometriose e as lesões endometrióticas intestinais possa nos conduzir a uma melhor compreensão da fisiopatologia desta doença. 
1. Kennedy S, Bergqvist A, Chapron C, D'Hooghe T, Dunselman G, Greb R, et al. ESHRE guideline for the diagnosis and treatment of endometriosis. Hum Reprod. 2005;20(10):2698-704.

2. Boling RO, Abbasi R, Ackerman G, Schipul AH Jr, Chaney SA. Disability from endometriosis in United States Army. J Reprod Med. 1988;33(1):49-52.

3. Cornillie FJ, Oosterlynck D, Lauweryns JM, Koninckx PR. Deeply infiltrating pelvic endometriosis: histology and clinical significance. Fertil Steril. 1990;53(6):978-83.

4. Chapron C, Chopin N, Borghese B, Foulot H, Dousset B, Vacher-Lavenu MC, et al. Deeply infiltrating endometriosis: pathogenetic implications of the anatomical distribution. Hum Reprod. 2006;21(7):1839-45.

5. Daraï E, Bazot M, Rouzier R, Houry S, Dubernard G. Outcome of laparoscopic colorectal resection for endometriosis. Curr Opin Obstet Gynecol. 2007; 19(4):308-13.

6. Anaf V, Simon P, El Nakadi I, Fayt I, Buxant F, Simonart T, et al. Relationship between endometriotic foci and nerves in rectovaginal endometriotic nodules. Hum Reprod. 2000;15(8):1744-50.

7. Remorgida V, Ragni N, Ferrero S, Anserini P, Torelli P, Fulcheri E. The involvement of the interstitial Cajal cells and the enteric nervous system in bowel endometriosis. Hum Reprod. 2005;20(1):264-71.

8. Anaf V, El Nakadi I, Simon P, Van de Stadt J, Fayt I, Simonart T, et al. Preferential infiltration of large bowel endometriosis along the nerves of the colon. Hum Reprod. 2004;19(4):996-1002.

9. Viganò P, Parazzini F, Somigliana E, Vercellini P. Endometriosis: epidemiology and aetiological factors. Best Pract Res Clin Obstet Gynaecol. 2004;18(2):177-200.

10. Bullimore DW. Endometriosis is sustained by tumour necrosis factor-alpha. Med Hypotheses. 2003;60(1):84-8.

11. Overton C, Fernandez-Shaw S, Hicks B, Barlow D, Starkey P. Peritoneal fluid cytokines and the relationship with endometriosis and pain. Hum Reprod. 1996; 11 (2):380-6.

12. Sofroniew MV, Howe CL, Mobley WC. Nerve growth factor signaling, neuroprotection, and neural repair. Annu Rev Neurosci. 2001;24:1217-81.

13. Schaible HG, Del Rosso A, Matucci-Cerinic M. Neurogenic aspects of inflammation. Rheum Dis Clin North Am. 2005;31 (1):77-101, ix.

14. Medina MG, Lebovic DI. Endometriosis-associated nerve fibers and pain. Acta Obstet Gynecol Scand. 2009;88(9):968-75.

15. Anaf V, Simon P, El Nakadi I, Fayt I, Simonart T, Buxant F, et al. Hyperalgesia, nerve infiltration and nerve growth factor expression in deep adenomyotic nodules, peritoneal and ovarian endometriosis. Hum Reprod. 2002;17(7): 1895-900.
16. Wang G, Tokushige N, Russell P, Dubinovsky S, Markham R, Fraser IS. Hyperinnervation in intestinal deep infiltrating endometriosis. J Minim Invasive Gynecol. 2009; 16(6):713-9.

17. White DM, Mansfield K. Vasoactive intestinal polypeptide and neuropeptide $Y$ act indirectly to increase neurite outgrowth of dissociated dorsal root ganglion cells. Neuroscience. 1996;73(3): 881-7.

18. Possover M, Tersiev P, Angelov DN. Comparative study of the neuropeptide-Y sympathetic nerves in endometriotic involved and noninvolved sacrouterine ligaments in women with pelvic endometriosis. J Minim Invasive Gynecol. 2009;16(3):340-3.

19. Kelm Junior AR, Lancellotti CL, Donadio N, Auge AP, Lima SM, Aoki $T$, et al. Nerve fibers in uterosacral ligaments of women with deep infiltrating endometriosis. J Reprod Immunol. 2008;79(1):93-9.

20. Revised American Society for Reproductive Medicine classification of endometriosis: 1996. Fertil Steril. 1997;67(5):817-21.

21. Tokushige N, Markham R, Russell P, Fraser IS. High density of small nerve fibres in the functional layer of the endometrium in women with endometriosis. Hum Reprod. 2006;21 (3):782-7.

22. Tokushige N, Markham R, Russell P, Fraser IS. Nerve fibres in peritoneal endometriosis. Hum Reprod. 2006;21(1 1):3001-7.

23. Berkley KJ, Dmitrieva N, Curtis KS, Papka RE. Innervation of ectopic endometrium in a rat model of endometriosis. Proc Natl Acad Sci USA. 2004; 101(30): 1 1094-8.

24. Barcena de Arellano ML, Arnold J, Vercellino F, Chiantera V, Schneider A, Mechsner S. Overexpression of nerve growth factor in peritoneal fluid from women with endometriosis may promote neurite outgrowth in endometriotic lesions. Fertil Steril. $2011 ; 95(3): 1123-6$

25. Barcena de Arellano ML, Arnold J, Vercellino GF, Chiantera V, Ebert AD, Schneider A, et al. Influence of nerve growth factor in endometriosis-associated symptoms. Reprod Sci. 2011;18(12): 1202-10.

26. Lv D, Song H, Shi G. Anti-TNF-alpha treatment for pelvic pain associated with endometriosis. Cochrane Database Syst Rev. 2010;(3):CD008088.

27. Ribeiro PA, Rodrigues FC, Kehdi IP, Rossini L, Abdalla HS, Donadio $\mathrm{N}$, et al. Laparoscopic resection of intestinal endometriosis: a 5-year experience. J Minim Invasive Gynecol. 2006; 13(5):442-6.

28. Ferrero S, Haas S, Remorgida V, Camerini G, Fulcheri E, Ragni N, et al. Loss of sympathetic nerve fibers in intestinal endometriosis. Fertil Steril. 2010;94(7):2817-9.

29. Possover M, Quakernack J, Chiantera V. The LANN technique to reduce the postoperative functional morbidity in laparoscopic radical pelvic surgery. J Am Coll Surg. 2005;201 (6):913-7. 